\title{
Routing Analysis of Wireless Ad Hoc Network Based on Mobile Node Technology
}

\author{
WeiChun ${ }^{1}$, LiMing ${ }^{2}$ and LiuZhigang ${ }^{3, a}$ \\ ${ }^{1}$ School of Electronic and Information Engineering,Wuhan Donghu University, Wuhan 430211, \\ China; \\ ${ }^{2}$ Information management center, Air Force Early Warning Academy, Wuhan 430019,China; \\ ${ }^{3}$ Nanchang Institute of Science \& Technology, Nanchang 330108,China ) \\ aliuzhigang_777@qq.com
}

Key words: mobile node; Ad hoc Network; ant colony algorithm; NS2 simulation; average hops

\begin{abstract}
It leads to the uncertainty of the network topology that the random mobility of nodes in mobile Ad hoc networks. In order to accurately predict network topology performance, and effectively detect ad hoc network efficiency, capacity and stability, it has proposed in ad hoc network mobile node optimization method based on Ant Colony Algorithm. In order to verify the feasibility of the method, the hoc Ad network is built using NS2, and the performance of the network's successful receiving rate, the receiving end delay and the average hops is detected by the simulation calculation. By the final test results, it is found that using ant colony algorithm can effectively improve the successful network receiving rate, and reduce the average delay of the receiver and the average number of hops, which can effectively improve the stability of mobile node routing link.
\end{abstract}

\section{Introduction}

With the rapid development of wireless communication technology and driven by more powerful function of wireless devices and application software, the development prospect of wireless communication has been remarkable changed, the rise of the new fourth generation mobile communication technology has greatly improved the data transmission rate. With the continuous deepening of the concept and application of the Internet of things, wireless multi hop mobile Ad hoc network has attracted more and more researchers' attention. Mobile Ad hoc networks is expected to will become the key part of the future 4G network and the Internet of things, and it is hoped that the wireless multi hop Ad hoc network performance can become an important part of the overall function of next generation wireless communication network, so it has important significance to study its performance.

\section{Ad hoc network based on mobile node}

Hoc Ad network is a self-organizing network, with wireless multi hop, the network can use these nodes to find routing and communication, and have no need to use other infrastructure, which is based on mobile node technology. In ad hoc networks, the arbitrary node mobility caused the wireless topology network is not stable, and the routing performance is hard to predict, the network in addition can be run separately, can also cover a wide range of the Internet network. In general, 
the routing of mobile nodes includes several links, and the network example graph is shown in Figure 1.

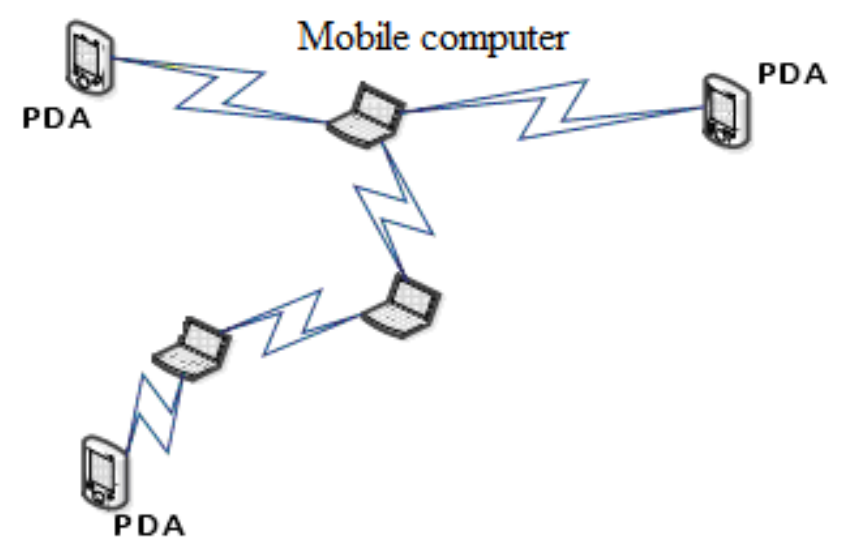

Fig. 1 Ad hoc network based on mobile node

Figure 1 shows the Ad hoc network based on the mobile node, its application value is very important. Different from the traditional wireless network and wired network, Ad hoc network does not require infrastructure, the mobile nodes can be taken as a medium of communication, the mobile performance of multi hop routing is strong, so the routing protocol is most important and is also the most difficult problem to be solved. In the movement process of node, the energy of the node is limited. When the energy consumption of the node, it will lead to the failure of the route, and then the communication is interrupted. So, when the energy consumption of nodes at a certain time of data communication, routing may fail at the next time, so we have to reestablish the link, which can cause the communication overhead and data packet loss. Therefore, it is significant important for the normal operation of the network that the research of routing energy, capacity and stability. Generally speaking, there are two issues are the most important in the study of network stability, which will be covered respectively as follows:

(1) The establishment of mobile node routing best can average distribute the whole network energy, which can screen out lower energy nodes and increase the network lifetime, and make data transmission more efficient, and improve the quality of network communication.

(2) The better routing strategy should be selected, the communication criterion of each hop should be determine, and the equalization method is be used to make the route with better stability and lower delay characteristics.

With the continuous upgrading of network, network performance requirements for video and voice transmission are constantly improving, so the network delay requirements is higher. Therefore, these problems must be taken into account if you want to ensure efficient and reliable operation of the Ad hoc network, which can be solved by the study of the mobile node routing.

\section{Ant colony optimization algorithm of Ad hoc network routing stability based on mobile nodes}

When mobile nodes discovery route, the basis of node response is the partitioning set, after the upstream node is stable, the response of the routing request packet can be carried out, and then the packet is forwarded, otherwise unable to forward data, so that the network is not stable, the link stability model is shown in Figure 2. 


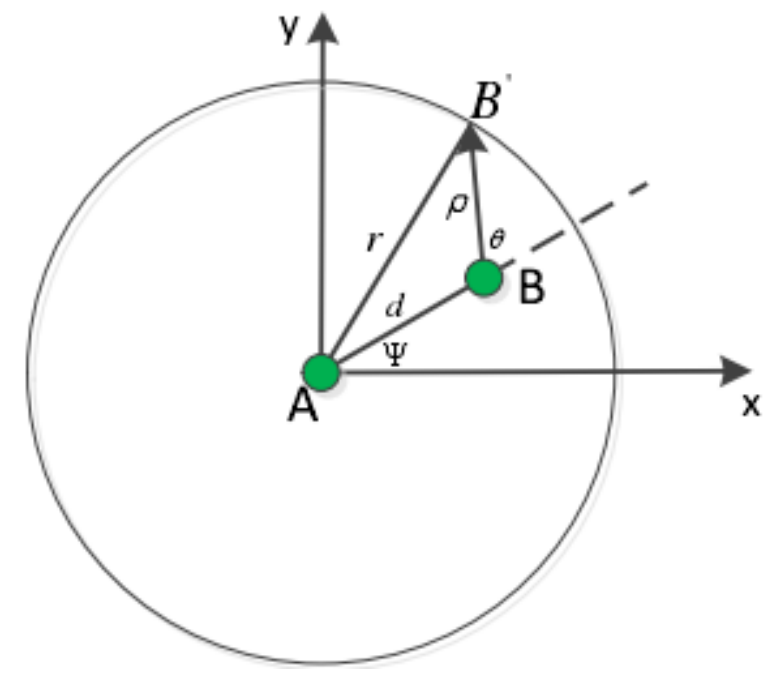

Fig. 2 The stability estimation model of mobile node routing link

Figure 2 shows the stability estimation model of mobile node routing link, between node $\mathrm{A}$ and $\mathrm{B}$, when $\mathrm{B}$ receives a data packet sent by $\mathrm{A}$, if within the stability range of $\mathrm{A}$ data transmission, the stability will be estimated. We refer to $\mathrm{A}$ as a reference point, $\mathrm{B}$ moves with certain speed $\mathrm{V}$ relative to A, the movement time is $t$. Suppose then node B just is in the scope of A, in this paragraph of time the trajectory of $\mathrm{B}$ is $\rho$, according to the cosine theorem we can get:

$$
\begin{aligned}
r^{2}= & \rho^{2}+d^{2}-2 \rho d \cos (\rho-\theta) \\
& (\rho+d \cos \theta)^{2}+d^{2} \sin ^{2} \theta
\end{aligned}
$$

After finishing the formula (1), we can get the formula (2) as following.

$$
\rho(d, \theta)=\sqrt{r^{2}-d^{2} \sin ^{2} \theta}-d \cos \theta
$$

Assuming at the moment $t_{0}$, the coordinates of $\mathrm{A}$ is $\left(x_{A}\left(t_{0}\right), y_{A}\left(t_{0}\right)\right)$, the coordinates of $\mathrm{B}$ is $\left(x_{B}\left(t_{0}\right), y_{B}\left(t_{0}\right)\right)$, the distance between $\mathrm{A}$ and $\mathrm{B}$ at the time is:

$$
d\left(t_{0}\right)=\sqrt{\left(x_{A}\left(t_{0}\right)-x_{B}\left(t_{0}\right)\right)^{2}+\left(y_{A}\left(t_{0}\right)-y_{B}\left(t_{0}\right)\right)^{2}}
$$

Ant colony algorithm is generally taken to solve complex combinatorial problem, and mobile node routing link is also belongs to complex combinatorial optimization problem, Ad hoc network model can be established by $m$ nodes. Suppose the number of ants is $n, d_{i j}(i, j=1,2, \ldots \ldots, m)$ is the distance between mobile nodes $i, j, \tau_{i j}$ is the pheromone concentration of nodes $i, j$. At the initial time, the pheromone concentration of network is the same, with the passage of time, the nodes energy can be consumed and pheromone concentration begins to be volatile, which similar with ants leave, using $1-\rho$ to show the degree of volatilization. After $m$ moment, ants have completed the time cycle, the pheromone concentration in the path is shown as formula (4).

$$
\begin{aligned}
& \Delta \tau_{i j}{ }^{k}=Q / d_{i j} \quad \text { if the } k \text { th ant uses edge }(i, j) \\
& \Delta \tau_{i j}{ }^{k}=0, \quad \text { otherwise }
\end{aligned}
$$


Where $Q$ is a constant, which is the distance between nodes $i$ and $j$, when $k$ ants through the path, the pheromone concentration is increased, otherwise the pheromone concentration is constant. The algorithm can completed by many ants, and each ant have independent search solution, so that the network can be optimized.

\section{Simulation of Ad-Hoc routing protocol based on NS2}

NS2 is a professional network simulation tool, which is oriented to the prior driven object, and provides for users with the programming and network configuration tool. It is the extension of TCL language. NS command can be used to define the Ad-Hoc network, topology, capacity, and receiving and sending of data statistics. NS2 has dynamic data observation function, and configuration of the graphics display, simulation network and data can be graphically displayed, the network established by the simulation is shown in Figure 3.

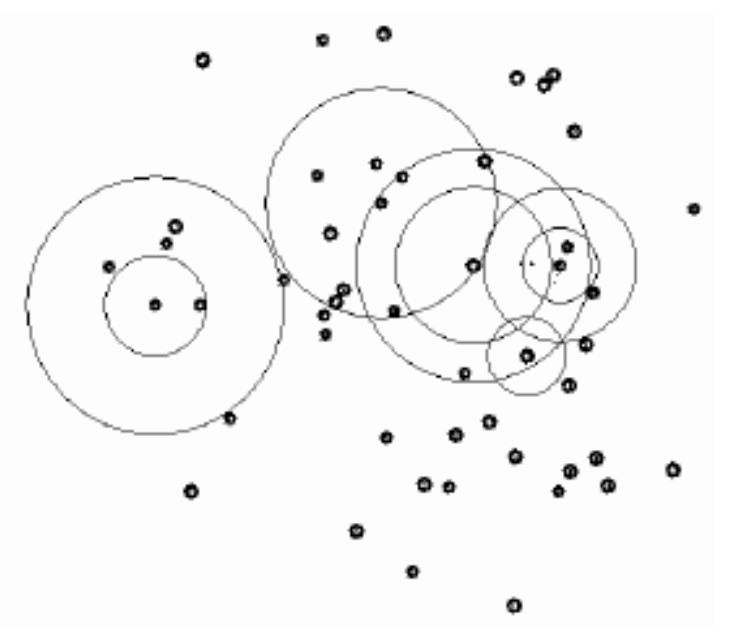

Fig.3 NS2 simulation network

Figure 3 shows the schematic diagram of the simulation network, the scale of the scene is $1200 \mathrm{~m} \times 1200 \mathrm{~m}$, there are 50 mobile nodes in the scene, the transmission rate is $2 \mathrm{Mbit} / \mathrm{s}$, the average speed of the node is $0,1 \mathrm{~m} / \mathrm{s}, 5 \mathrm{~m} / \mathrm{s}, 10 \mathrm{~m} / \mathrm{s}$ respectively. The functional advantage of ant colony algorithm is verified by network simulation. The simulation mainly analysis successful reception rate of data packet, the receiver delay and average number of hops. The simulation results of successful reception rate, receiver delay are shown in Table 1 below.

Tab. 1 The simulation results of Ad-Hoc network

\begin{tabular}{|c|c|c|c|c|}
\hline Average velocity & $0 \mathrm{~m} / \mathrm{s}$ & $1 \mathrm{~m} / \mathrm{s}$ & $2 \mathrm{~m} / \mathrm{s}$ & $3 \mathrm{~m} / \mathrm{s}$ \\
\hline Receiving rate without ant colony algorithm (\%) & 69.28 & 70.12 & 60.25 & 50.33 \\
\hline Receiving rate with ant colony algorithm (\%) & 88.29 & 80.25 & 79.32 & 75.29 \\
\hline Receiver delay without ant colony algorithm (s) & 0.0325 & 0.0293 & 0.0528 & 0.0632 \\
\hline Receiver delay without ant colony algorithm (s) & 0.0112 & 0.0108 & 0.0325 & 0.0438 \\
\hline
\end{tabular}

Table 1 in the simulation of ad hoc network routing performance of the statistical results, by the statistical results can be seen, along with the increase of the speed of mobile node, the success of ad hoc network reception rate becomes smaller, the network latency increases, and the use of ant colony algorithm can be significantly improved network data receiving rate and reduce the delay of the terminal. 


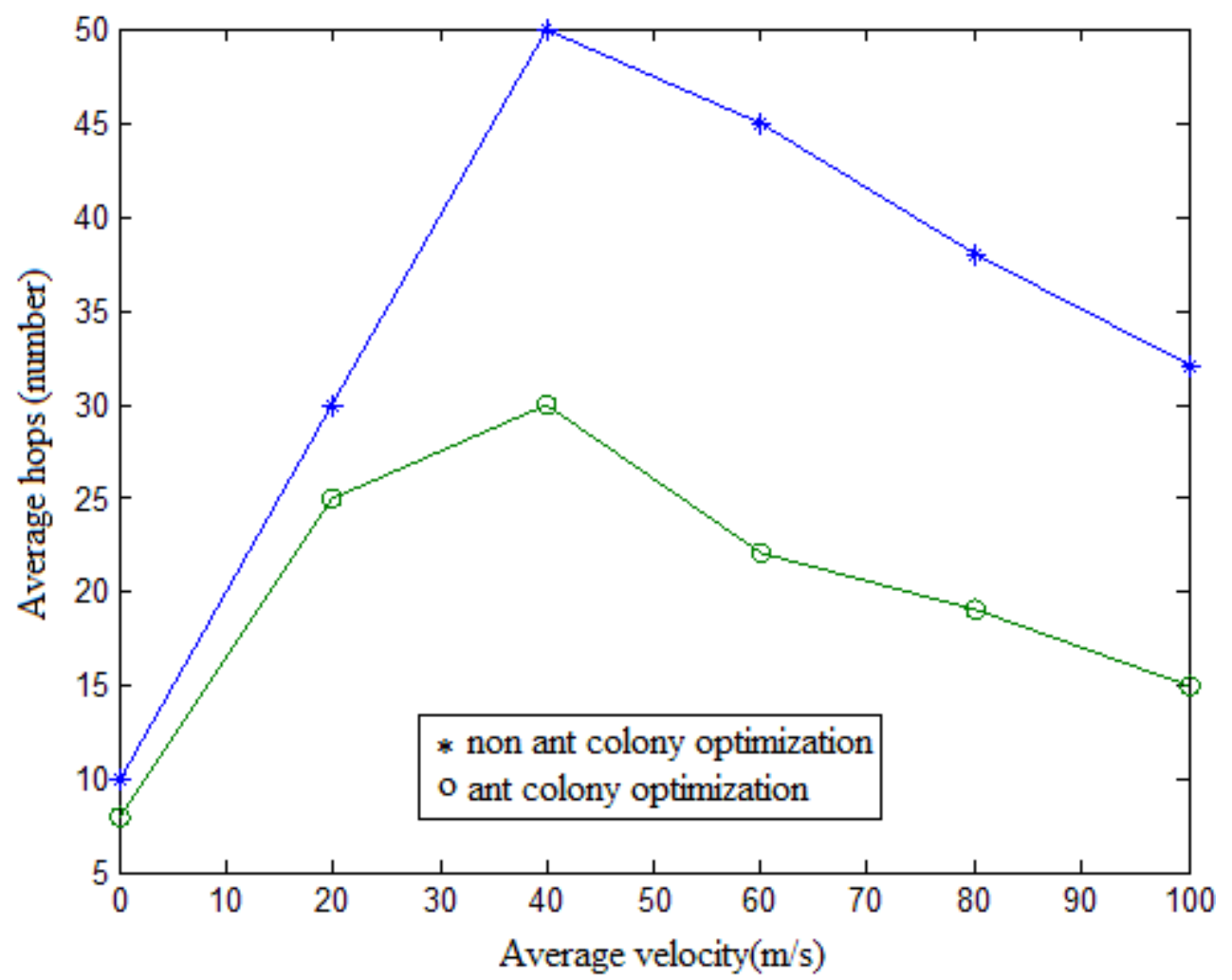

Fig.4 Average hops count under different moving speeds

Average number of hops generally refers to the mobile node number from the sending point to the receiving point. Figure 4 shows the average number of hops using ant colony algorithm and not using ant colony algorithm at different average velocity, it can be seen from the figure that using ant colony algorithm can effectively reduce the average number of hops, which can be more convenient to find the best route.

\section{Conclusions}

Because of the Ad hoc network is a flexible network that can be build anytime, anywhere, without infrastructure and pre-configured or management, it is realized that mobile Ad hoc networks can bring huge commercial potential and advantages. In this paper, the routing performance of mobile node Ad-Hoc network is studied, and the ant colony algorithm is used to optimize the design of the network. Finally, the optimization effect of the network is verified by using NS2, the experimental results show that using ant colony algorithm can improve the data receiving rate of the network and reduce the time delay of the terminal, which can improve the efficiency and stability of the whole network.

\section{Acknowledgments}

The work was supported by the scientific research planning project of Education Department of Hubei Province with the project number B2016317 and the project name Analysis of network capacity of wireless hoc ad networks.

The work was also supported by the Post Doctoral Orientation Funding Project of Yunnan Province in 2015. The work was also supported by the Project of Youth Science Foundation of National Natural Science Foundation with the project number 41505015 and the project name Measurement Method of Atmospheric High-resolution Microwave Radiometric Based on Virtual Mirror Antenna. Finally,the work was also supported by The science and technology research 
project of Education Department of Jiangxi Province in 2015 with the project number GJJ150156 and the project name Prediction of Forest Fire Spread Based on Grey GM $(1,1)$ Model.

\section{References}

[1] $\mathrm{Hu} \mathrm{Xi}$, Li Zhe, Liu Jun. A link stability prediction based on Demand routing protocol in mobile Ad hoc Networks [J]. Journal of Electronics \& Information Technology, 2010,32 (2): 284-289.

[2]P.SrinivasanandP.Kamalakkannan.REAQ-AODV:RouteStabilityandEnergyAwareQoSRoutingin MobileAdhocNetworks[C].IEEEICoAC'12,AnnaUniversity,Chennai, 2012: 1-5.

[3]Xia Hui, Jia Zhiping, Zhang Zhiyong, et al. A link stability prediction based Multicast routing protocol in mobile Ad hoc Networks [J]. Chinese Journal of Computer, 2013,36(5):926-935.

[4] Wang Qingwen, Shi Haoshan, Qi Qian. A new Ad hoc network cross layer energy balance broadcast protocol. Journal of Northwestern Polytechnical University [J].2011,29 (5): 671-675.

[5] Wu Kejun, Yu Quan, Tian Yanxia. A Novel location aided Ad Hoc on-demand vector routing protocol [J].Acta Electronica Sinica, 2010,38 (4): 983-988.

[6] Huang Guorui, Cao Xianbin, Wang Xufa. An Ant colony optimization algorithm based on pheromone diffusion[J]. Acta Electronica Sinica, 2005,32 (5):865-868

[7] Dong Xuewen, Ma Jianfeng, Niu Wensheng, et al. Attack analysis model of Ad Hoc security routing protocol based on strand space model[J]. Journal of Software, 2011,22 (7): 1641-1651.

[8] Li Qin, Zeng Qingkai. Verifying mobile Ad hoc security routing protocols with type inference[J]. Journal of Software, 2009, 20 (10): 2822-2833.

[9] Pu Baoxing, Yang Luming, Wang Weiping, Generation and Extension of linear network coding [J]. Journal of software, 2011,22 (3): 558-571.

[10] Fu Bin, Li Renfa, Liu Caiping, et al. A congestion aware routing protocol based on network coding in wireless sensor networks [J]. Journal of Computer Research and Development, 2011,48 (6): 991-999.

[11] Lu Yi, Xiao Song, Wu Chengke. opportunistic network coding based on delay-sensitive broadcast transmission algorithm [J] .Acta Electronica Sinica, 2011,39 (5): 1214-1219.

[12] Pu Baoxing Wang Weiping, Evaluation and analysis of the computation cost of linear network coding [J]. Journal on communications, 2011,32 (5): 47-55.

[13]Wang Wei, Yu Li, Zhu Guangxi, Li Hui. A Novel transmission scheme of Multiple cognitive relay based on network coding [J]. Journal of Electronics \& Information Technology, 2011,33 (4): 869-873.

[14] Jiao Xianlong, Wang Xiaodong, Zhou Xingming. Network coding method Using heuristic algorithm for wireless Ad Hoc Networks[J]. Journal of software, 2010,21 (11): 2892-2905.

[15] Chen Guihai, Li Hongxing, Han Song, et al. Network coding-aware Multipath routing in multi-hop networks [J], Journal of Software, 2010,21 (8): 1908-1919.

[16] Wang Xiaodong, Huo Guangcheng, Sun Haiyan. An opportunistic routing for MANET based on partial network coding [J]. Acta Electronica Sinica, 2010,38 (8): 1736-1740. 